\title{
An Approach for Scratch Removal from Digital Image using Image Reconstruction
}

\author{
Md. Iqbal \\ Quraishi \\ Dept. of Information \\ Technology \\ Kalyani Govt. Engg. \\ College, Nadia, India
}

\author{
Mallike De \\ Dept. of Engg. and \\ Tech. Studies \\ University of Kalyani \\ Kalyani, Nadia, India
}

\author{
Goutam Das \\ Dept. of Information \\ Technology \\ Kalyani Govt. \\ Engineering College \\ Kalyani, Nadia, India
}

\author{
Abhishek \\ Srivastava \\ Dept. of Information \\ Technology \\ Kalyani Govt. Engg. \\ College, Nadia, India
}

\begin{abstract}
Old digital pictures or scanned images of old paper based images tend to have annoying scratches. This effects the overall quality of the image, both quantitively and visually. To remove these scratches and to generate a perfect noise and scratch free image, a novel method is presented in this paper. This approach not only detects scratches in an image but also removes them effectively. The method starts with a scratched digital version of an image then it removes noises after which region of scratch are identified. Filtering is an important step in this proposed method. Here filtering is a two level process after which a specific superimposing method is been applied to reconstruct the scratch and noise free image.
\end{abstract}

\section{General Terms}

Image processing, Artificial Intelligence.

\section{Keywords}

Image scratch, digital image processing, noise, superimposing, image reconstruction, adaptive median filter, non linear filter, region of interest

\section{INTRODUCTION}

The research on removal of scratches in digital images can be traced back to the Joyeux, Buisson, Besserer and Boukir's work in [1]. They used a method for line scratches detection strengthened by a Kalman Filter, This detector is based on morphological transformations and lot of researchers was done in this area. Francesco Isgro and Domenico Tegolo implemented a method for scratch restoration on a distributed system in [3], it work as an optimization problem and it is solved by an ad hoc genetic algorithm. But further work needs to improve the fitness function to refine the image restoration. The Experiment of this paper gives a good result. Ruth Bergman, Ron Maurer, Hila Nachlieli, Gitit Ruckenstein, Patrick Chase and Darryl Greig were presented a comprehensive set of algorithm for the removal of scratches from digital images in [4]. They categories those algorithm in several aspects like making soft vs. hard classification of pixels, using local vs. non-local information, and taking advantage of external assistance. Anil N. Hirani and Takashi Totsuka developed an algorithm for noise and scratch removal from image in [5]. Algorithm is based on a novel, hierarchical and prototype based interactive application of the theory of projection onto convex sets (POCS) using a effective closed convex set and projection. They mainly apply frequency and spatial domain constraint in the input image. Kenta Chishima and Kaoru Arakawa used an adaptive median filter with a variant window to removed line scratches in [6]. This is used to detect straight line element around scratches using Hough transform. They used the combination of this filter with scratch detection, film scratches are effectively removed, without making deformation of image components. T. Bretschneider, O. Kao used a technique in [7], which highlights the physical structure of the scratch to distinguish between an actual scratch and an image feature. It also used the presence of dominant features parallel to the scratch. Here used an iterative interpolation to reconstruct the affected pixels and the convergence of the algorithm is guaranteed for noise remove. Laurent Joyeux, Samia Boukir, Bernard Besserer used a detector and line scratch removal algorithm is an efficient Bayesian technique in [8]. Where the detector is based on an 1D-extrema localization and a Kalman filter. They used a simple polynomial interpolation to reconstruct the low frequencies of the deteriorated areas and higher frequencies using a MAP reconstruction approach. T. Bretschneider, O. Kao and P.J. Bones used the discrete wavelet decomposition technique to remove the vertical scratch in digitized film sequence in [11]. This transformation splits the image into approximation and detail coefficients. The algorithm reconstructs the region of the scratch and finally the synthesis of the wavelet coefficients generates a restored version of the scratched image frame. M.Haindl and S.Simberova also done a research on Scratch Removal method .they used algorithm based on adaptive multidimensional prediction in [12]. Here predictor parameters cannot be directly identified so a special approximation is introduced. Domenico Tegolo and Francesco Isgr'o has presented an application of analysis of the statistics of the grey levels, genetic algorithm in [13].Here used an approached as an optimization problem to remove the scratches, and it is solved by using genetic algorithm. This scratch detection differs from classical approaches in the use of the distribution of the column variance of the grey levels, which allows a more robust detection. O. Kao, J. Engehausen has presented a novel algorithm for detection and removal of scratches in [14].They used two-phase approach for reconstructing the destroyed image section without affecting the rest of the image. The first phase include scratch detection based on an adaptive binarisation, the second phase includes luminance smoothing process and application of a modified vector median filter V. Bruni, D. Vitulano, A. Kokaram in [15] has presented a fast and effective algorithm for removing line scratches in old movies. The algorithm uses Weber's law, the experimental results are very satisfying and the image is completely recovered without local artifacts or annoying smoothing effects. Lucia Maddalena and Alfredo Pestrosino in [16] have presented the problem of detecting and removing 
blue scratches from digital image sequences. They proposed two algorithms firstly BSD (blue scratch detection) for the detection of blue scratches in a digital image and secondly BSR (blue scratch removal) algorithm includes reconstructing corrupted information in the defected area. The Experiment of this paper produces a good result. Robin D. Morris, W. J. Fitxgerald and A. C. Kokaram in [17] has presented the problem of detecting and subsequently removing line scratch in motion pictures. They used a sampling algorithm based on the Reversible Jump Markov chain Monte Carlo (MCMC) Framework which determines the number of line present. The detection algorithm proved accurate in determining the number as well as location of line scratches. Markus Gla, Birgit oller, Anne Zirkel, Kristin Wachter, Stefan Huttelmaier and Stefan Posch present an automatic analysis pipeline detecting scratch boundaries and measuring areas based on level sets in[18]. They extend non-PDE level sets for topology-preservation and use an entropy-based energy functional. By this approach they design segments in every image, and then they used vector machines to identify images showing no scratch. Mehdi Haji, Tien D Bui and Ching Y. Suen we formulate the noise removal and recognition as a single optimization problem in [19] which can be solved by expectation maximization and recognition engine that is trained for clean images. They used fuzzy inference systems in the initialization step of the optimization process. Kyung-tai Kim, Eun Yi Kim defined the texture and shape properties from spatial domain and use these for scratch detection in [20]. There method which involves two procedures. One is divided the input image into scratches and non-scratches using a neural network (NN)-based texture classifier and another is removal of some false alarms by shape filtering using a morphological filter with new structuring elements which based on the shape characteristics of scratches. M. Kemal Güllü, Oğuzhan Urhan, Sarp Ertürk in [25] has presented a paper for detecting and removing scratch for archive film sequences. They proposed a detection which exploits both spatial and temporal information. Spatial information is used to obtain candidate scratch while temporal information is used to verify the coherency of the candidate. Kamon Homkajorn, Mahasak Ketcham, and Sartid Vongpradhip in[26] has presented a paper which involves removing scratches or damages that exists on QR-code. The applied procedure consists of Segmentation by HSV, Dilation, Binary image and noise reduction by Median Filter. The paper is effective and produces good results. Bernard Besserer and Cedric Thire in [27] has presented a paper on detection and tracking for line scratches removal in image. The method they used includes a input projection of each image then 1Dextrema detector provides candidate after that a MHT (Multiple Hypothesis Tracker) uses these candidates to create and keep multiple hypothesis. The proposed method gives good result. Laurent Joyeux, Samia Boukir, Bernard Besserer in[28] has presented a detection and reconstruction approach for removing line scratches from degraded motion picture films. They used kalman filter for tracking the potential lines over the frames after that a new Bayesian restoration technique with both low and high frequencies around and inside the detected deteriorations is investigated which reconstruct the damaged areas. Alexandre Ulisses Silva,Luís Corte-Real in[29] has presented a paper for removing line scratches and blotches from flims or video sequences. they used a method named as digital restoration chain. The basic element in this chain consists of digital encoder, artifact generator, interpolators and quality measurer with each element having a specific function. The algorithm they have used is very fast and effective. Timothy K. Shih, Louis H. Lin, Wonjun Lee ion [30] has presented detection and removal technique of long scratches in
Aged films .They proposed a new approach based on two strategies, the strategies are subdivision of video bands and progressive detection in painting. The proposed solution is flexible and more than $86 \%$ of the effective defects are detected and removed.

Our proposed system which works on digital old images or scanned images of old photographs with visual scratches and various types of noises. The proposed system implements two levels of image filtering. Filtering is the only way found in digital image processing that can remove different types of noises. But to remove scratch generally found filters are not effective. Before removing scratch, the scratch needs to be identified and recognized. Our proposed method identifies and uses a specific superimposing technique to remove scratches from an image. Reconstruction of the image as in a scratch free condition is the objective of this work.

\section{IMPLEMENTATION:}

\subsection{Flow diagram of the proposed system}

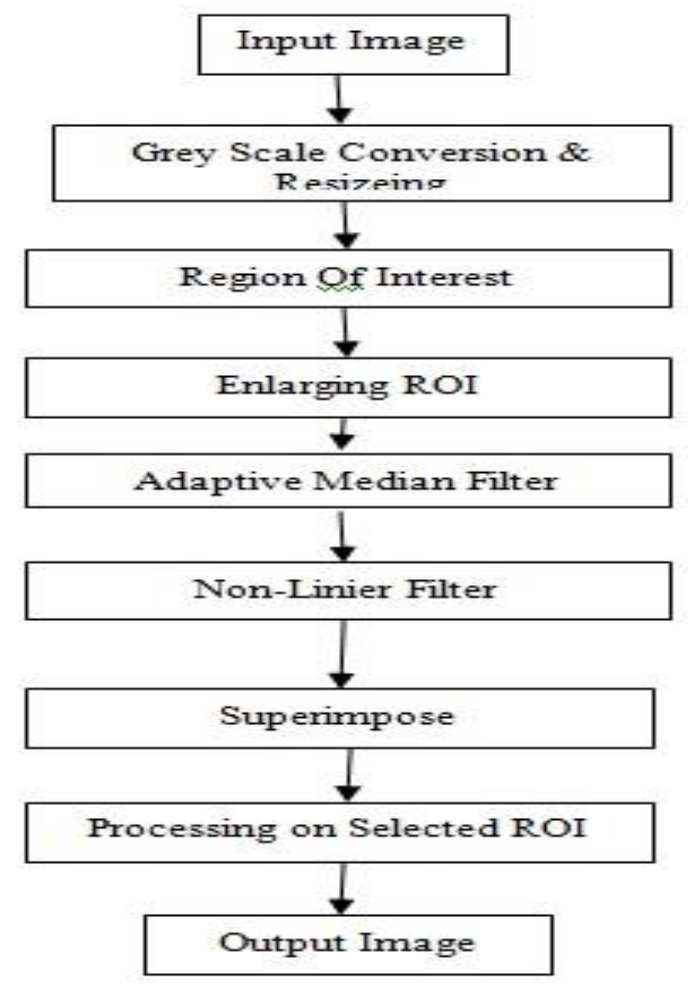

\subsection{Input Image:}

The input image is taken in RGB scale shown in Fig-1.

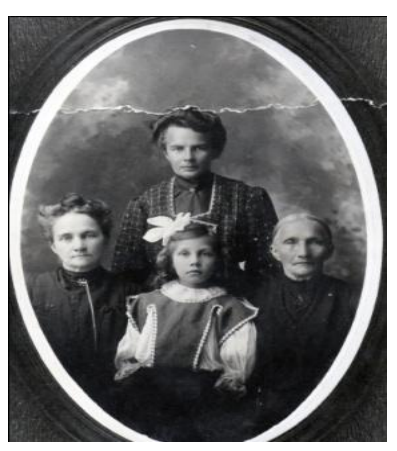

Fig-1 


\subsection{Grey Scale Conversions \& Resizing:}

The input image is converted into grey scale and resized into 256x256 pixels shown in Fig-2.

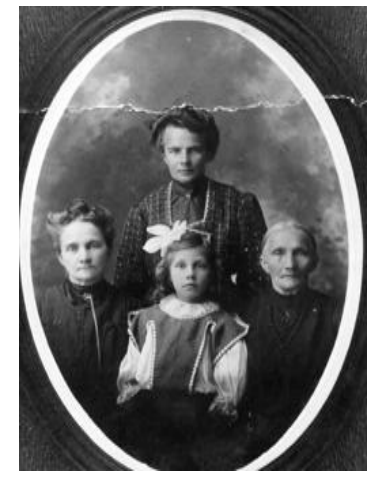

Fig-2

\subsection{Region Of Interest:}

The Region of Interest (ROI) has been selected from the acquired image which has been shown in Fig-3

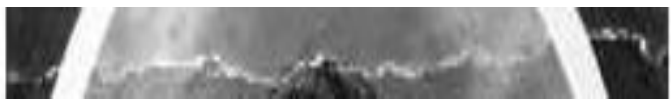

Fig-3

\subsection{Enlarging ROI:}

The Region of Interest is enlarged shown in fig-4.

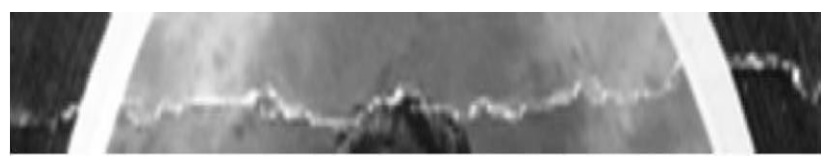

Fig-4

\subsection{Filtering:}

\subsubsection{Adaptive Median Filter:}

In this step Adaptive Median Filter have been applied to the enlarged image which makes the image smoother and clear which has been shown in Fig-5

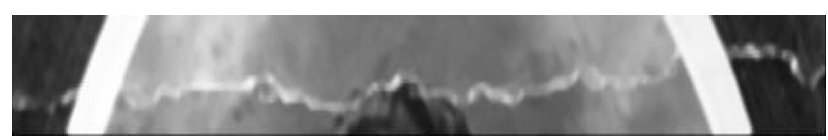

Fig-5

\subsubsection{Non-Linier Filter:}

Three-phase harmonic filters are shunt elements that are used in power systems for decreasing voltage distortion and for power factor correction. Nonlinear elements such as power electronic converters generate harmonic currents or harmonic voltages, which are injected into power system. It enhances the image smoother as a compared to the previous one shown in Fig-6

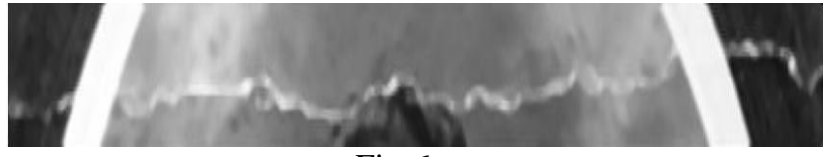

Fig-6

\subsection{Superimpose:}

The output of the non linier filter is placed on the resized image and the final output image is shown in fig-7

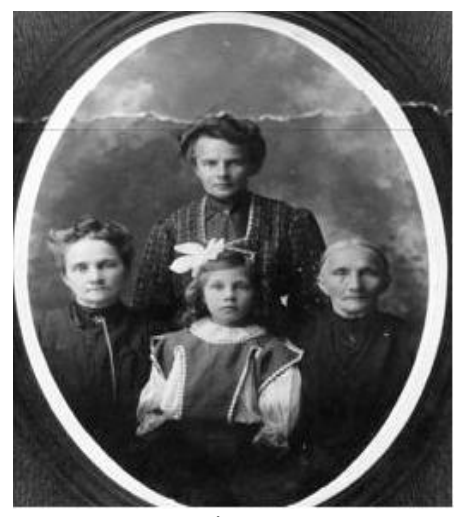

Fig-7

\subsection{Processing on Selected ROI:}

A selected pixel value is taken from the image and placed on the selected ROI pixel and the final output image is shown in Fig-8.

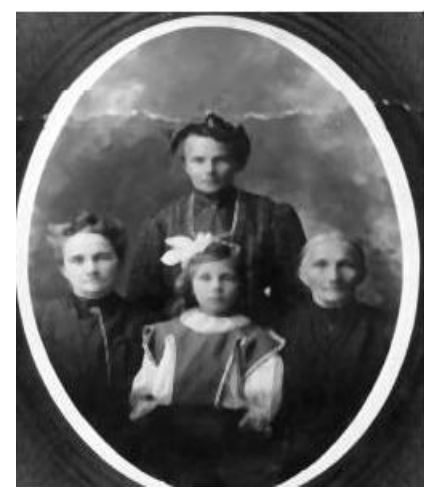

\section{CONCLUSION}

A novel method to remove scratches from an digital image is shown in this paper. The approach is a multi level filtered image reconstruction. The procedure involves Adaptive median filter and non linier filter as a two level filtering approach. Implementing superimposing technique to reconstruct the image in a specified manner, to generate the desired scratch free image. Using superimposing the selected pixels of the original image is placed in the selected ROI. The proposed method produces overall effective visual results. A further work can be carried out to show its effectiveness in old digital videos.

\section{REFERENCES}

[1] L. Joyeux, O.Buisson, B. Besserer, S. Boukir. Detection and Removal of Line Scratches in Motion Picture Films. University de La Rochelle, avenue Marillac,France

[2] R.Thilepa, M.Thanikachalam. A Paper On Automatic Fabrics Fault Processing Using Image Processing 
Technique In MATLAB. An International Journal(SIPIJ) Vol.1, No.2, December 2010

[3] Francesco Isgro, Domenico Tegolo. Restoration of vertical line scratches with a distributed genetic algorithm.

[4] Ruth Bergman, Ron Maurer, Hila Nachlieli, Gitit Ruckenstein, Patrick Chase, Darryl Greig. Comprehensive Solutions for Removal of Dust and Scratches from Images. HP Laboratories Israel HPL2007-20 February 6, 2007

[5] Anil N. Hirani, Takashi Totsuka. A Projection Based Algorithm for Scratch and Wire Removal in Images. Hashimoto Signal Processing Lab.Sony Research Center, Tokyo

[6] Kenta Chishima, Kaoru Arakawa. A Method of Scratch Removal from Old Movie Film Using Variant Window by Hough Transform. Department of Computer Science, Graduate School of Science and Technology

[7] T. Bretschneider, O. Kao. Detection and Removal of Scratches in Digitised Film Sequences.Department of Computer Science, Technical University of Clausthal,Julius-Albert-Straße 4, 38678 Clausthal, Germany

[8] Laurent Joyeux, Samia Boukir, Bernard Besserer. Film Line Scratch Removal Using Kalman Filtering and Bayesian Restoration. WACV'2000, IEEE Workshop on the Application of Computer Vision, Palm Springs, California, December 2000

[9] Anil Kokaram.Detection And Removal Of Line Scratches In Degraded Motion Picture Sequences.Engineering Department, university of Cambridge

[10] Michal Haindl \& Jiri Filip. Fast Restoration of Colour Movie Scratches. Institute of Information Theory and Automation Academy of Sciences of the Czech Republic

[11] T. Bretschneider ,P.J. Bones. Removal of Vertical Scratchesin Digitised Historical Film Sequences Using Wavelet Decomposition. Department of Electrical and Electronic Engineering

[12] M.Haindl. A scratch removal .Method.Institute of Information Theory and Automation, Czech Republic

[13] Domenico Tegolo. Scratch Detection And Removal From Static Images Using Simple Statistics And Genetic Algorithm. Universit a di Palermo

[14] O. Kao, J. Engehausen. Scratch removal in digitized film sequences. Department of computer Science, Germany

[15] V. Bruni, D. Vitulano. Fast Removal of Line Scratches in Old Movies. Istituto per le Applicazioni del Calcolo, Italy

[16] Lucia Maddalena, Alfredo Petrosino. Restoration of blue scratches in digital image sequences. Received 24 January 2006; accepted 28 April 2006

[17] Robin D. Morris. A Sampling Based Approach To Line Scratch Removal From Motion Pictures Frames. 2004, route des Lucioles, BP93, 06904 Sophia Antipolis, France.

[18] Markus Glaß, BirgitM“ oller, AnneZirkel, KristinW"achter, StefanH" uttelmaier and StefanPosch.
Cell migration analysis: Segmenting scratch assay images with level sets and support vector machines(Zentrumf ur

AngewandteMedizinischeundHumanbiologischeForschu ng(ZAMED),MartinLutherUniversityHalle

Wittenberg,Heinrich-Damerow-Str.1,06120

Halle(Saale), Germany b Institute

ofComputerScience,MartinLutherUniversityHalle-

Wittenberg,Von-Seckendorff

Platz1,06099Halle(Saale),Germany)

[19] Mehdi Haji, TienD.Bui,ChingY.Suen. Removal of noise patterns in handwritten images using expectation maximization and fuzzy inference systems(Department of Computer Science and Software Engineering, Concordia University, Montreal, Canada)

[20] Kyung-tai Kim, Eun Yi Kim. Film line scratch detection using texture and shape information(Department of Advanced Technology Fusion, Konkuk University, 1 Hwayang-dong, Gwangjin-gu, Seoul 143-701, South Korea)

[21] L.joyeux,S.Boukir.Reconstruction of degraded image sequences.Application to film restoration.(Received 16th April 1999,revised 29th sept 2000.accepted 30th sept 2000).

[22] Celia A. Zorzo Barcelos , Marcos Aurélio Batista. Image restoration using digital inpainting and noise removal(Received 26 May 2004; received in revised form 4 August 2005; accepted 18 December 2005).

[23] Ali Gangala, Temel Kayikcioglu, Bekir Dizdaroglu. An improved motion-compensated restoration method for damaged color motion picture films.( Received 27 May 2003; received in revised form 18 November 2003; accepted 20 January 2004).

[24] P. Pankajakshan , V. Kumar. Detail-preserving image information restoration guided by SVM based noise mapping(Department of Electrical Engineering, Texas A\&M University, College Station, TX 77843, USA Department of Electrical Engineering, Indian Institute of Technology Roorkee, Roorkee 247667, Uttaranchal, India, Available online 12 December 2006 Abstract)

[25] M. Kemal Güllü, Oğuzhan Urhan, Sarp Ertürk. Scratch Detection via Temporal Coherency Analysis and Removal using Edge Priority Based Interpolation(Department of Electronics and Telecommunications Engineering University of Kocaeli Kocaeli, Turkey \{kemalg, urhano, sertur\}@kou.edu.tr).

[26] Kamon Homkajorn, Mahasak Ketcham, and Sartid Vongpradhip. A Technique to Remove Scratches from QR Code Images (International Conference on Computer and Communication Technologies (ICCCT'2012) May 26-27, 2012 Phuket)

[27] Bernard Besserer and Cedric Thir. Detection and Tracking Scheme for Line Scratch Removal in an Image Sequence(Laboratoire Informatique, Image, Interaction (L3i), Universit'e La Rochelle, 17042 La Rochelle cedex 1, France \{bernard.besserer,cedric.thire\}@univ-lr.fr http://www.univ-lr.fr).

[28] Laurent Joyeux, Samia Boukir, Bernard Besserer. Tracking and MAP reconstruction of line scratches in degraded motion pictures(Laboratoire d'Informatique et d'Imagerie Industrielle (L3i), Universit'e de La Rochelle, 
avenue Michel Cr'epeau, 17042 La Rochelle cedex 1, France; e-mail: \{ljoyeux,sboukir,bbessere\}@univ-lr.fr Accepted: 13 August 2001).

[29] Alexandre Ulisses Silva , Luís Corte-Real Inesc-Porto. Removal of Blotches And Line Scratches From Film Aan Video Sequences Using a Digital Restoration Chain(Faculdade de Engenharia da Universidade do
Porto / INESC-Porto Rua dos Bragas 4099 Porto Codex, Portugal email:lreal@inescn.pt).

[30] Timothy K. Shih and Louis H. Lin. Detection And Removal Of Long Scratch Lines In Aged Films (Department of Comp. Sci. and Information Engineering Tamkang University, Taiwan tshih@cs.tku.edu.tw) 